\title{
COMPARATIVE STUDY BETWEEN MINILAPAROTOMY HYSTERECTOMY AND LAPAROSCOPIC HYSTERECTOMY FOR BENIGN UTERINE LESIONS
}

\author{
Abdel Moneim A. Saleh ; Azza A. Ibrahem ${ }^{a}$; Tarek M. El-Behidy ${ }^{a}$ and Amr A. El-Nemr ${ }^{b}$ \\ ${ }^{a}$ Professor of Obstetrics and Gynecology, Faculty of Medicine, Zagazig Unviersity
}

\begin{abstract}
Objective: The aim of the work is to investigate whether minilaparotomy hysterectomy for benign uterine lesions might be a reasonable alternative to laparoscopic hysterectomy in terms of operative and postoperative short-term outcomes. Methods: 105 patients scheduled to undergo total hysterectomy for a benign uterine lesion were divided into 3 groups according to their selection of the method of intervention after counseling: Group A (35 patients) minilaparotomy hysterectomy using conventional sutures.Group B (35 patients) minilaparotomy hysterectomy using bipolar vessel sealing system (Ligasure).Group C (35 patients) laparoscopic hysterectomy using Ligasure.

Result(s): The operating time in Group A (84.7 \pm 9.9 minutes), group B (55.3 \pm 7.8 minutes), while group C ( $94.8 \pm 16$ minutes).The duration until resumption of intestinal sounds in group $\mathrm{C}(12.3 \pm 2.5$ hours) in comparison to group $\mathrm{A}$ (17.4 \pm 1.9 hours) and group B (16.5 \pm 1.76 hours). Blood loss in group B $(99.1 \pm 30.8 \mathrm{ml})$, group A (130.3 $\pm 54.4 \mathrm{ml})$ and group C $(136.6 \pm 6.6 \mathrm{ml})$. The longest hospital stay occurred in group A (31.8 \pm 5.7 hours) versus groups B (20.7 \pm 2.5 hours) and $\mathrm{C}(19.3 \pm 6$ hours). The highest pain score was observed in group $\mathrm{A}(5.2 \pm 1.1)$ versus groups $\mathrm{B}(3.8 \pm$ 1.6) and $C(3.7 \pm 1.2)$. There was no significant difference in the incidence of intraoperative or postoperative complications.

Conclusion(s): The use of ligasure bipolar vessel sealing system in minilaparotomy hysterectomy was associated with less operative time and intraoperative blood loss, while it was comparable to laparoscopic hysterectomy in hospital stay and postoperative pain scoring with low morbidity and a short hospital stay. It may be a suitable alternative to laparoscopic hysterectomy which is ideal in areas which lack high laparoscopic experience or facilities.

Key Words: Minilaparotomy hysterectomy,Laparoscopic hysterectomy
\end{abstract}

\section{INTRODUCTION}

$\mathbf{H}$ ysterectomy is the second most frequently performed gynecologic procedure worldwide, second only to caesarean section. However, there is no universal agreement on the optimum method of hysterectomy. Hysterectomy can be performed by a number of different approaches like abdominal, vaginal and laparoscopic; each with its own merits and demerits. The optimum approach is generally guided by the indication for surgery, surgeon's training and preference, uterine size, presence and absence of associated pelvic pathologies and the patient's choice ${ }^{(\mathbf{1})}$.

Till recently, the choice of the route of hysterectomy was limited. Abdominal hysterectomy was performed for all benign and malignant gynecological disorders, while Vaginal Hysterectomy (VH) was reserved for prolapse cases. Lately, VH has been widely done for otherwise uncomplicated hysterectomies.

In the last two decades, laparoscopicAssisted Vaginal Hysterectomy (LAVH) and laparoscopic hysterectomy have become popular due to shorter hospital stay and minimal postoperative morbidity ${ }^{(2)}$.

Laparoscopic hysterectomy has disclosed new attractive horizons of minimally invasive surgery, offering both the surgeon and the patient excellent advantages in terms of intra- and postoperative results. In fact, patients undergoing operative laparoscopy have shown an excellent surgical outcome, shorter hospitalization, earlier recovery and an improved quality of life as compared to classical abdominal hysterectomy usually performed by a Pfannenstiel incision ${ }^{(3,4)}$.

Also, adhesions are less likely to form with laparoscopic surgery than with laparotomy possibly due to less direct peritoneal trauma and less exposure of peritoneal surface to air ${ }^{(5)}$.

However, laparoscopy has several limits, such as the induction of pneumoperitoneum which can cause serious intraoperative complications in patients with morbid obesity or with critical cardiopulmonary status. Laparoscopy needs a long learning curve, long operating time and more expensive equipment ${ }^{(6)}$.

Also, soft tissues, intramural myomas, or the inside of a hollow viscus cannot be palpated during laparoscopy ${ }^{(5)}$.

Laparotomic surgery has been improving and in this context, considering the enormous advantages of minimally invasive surgery, minilaparotomy has been used for decades for many benign gynecological conditions and also sterilization operations or its reversal and ovarian cystectomy operations with encouraging results ${ }^{(7)}$.

Minilaparotomy hysterectomy is defined as a hysterectomy done via a transverse abdominal incision into the upper level of the pubic hair no longer than $6 \mathrm{~cm}$ in length. This technique was firstly used by Dr. Marco A. Pelosi II in 1995 and it is said to combine the technical advantages of standard laparotomy (e.g. shorter learning curve) and the convalescent advantages of laparoscopy (e.g. low morbidity, short hospital stay and good 
cosmetic results). So, it could be an alternative to laparoscopic hysterectomy ${ }^{(\mathbf{8})}$.

The aim of the work is to investigate whether minilaparotomy hysterectomy for benign uterine lesions might be a reasonable alternative to laparoscopic hysterectomy in terms of operative and postoperative short-term outcomes.

PATIENTS AND METHODS

This prospective non-randomized study was conducted in the Department of Obstetrics and Gynecology and the Cytogenetic \& Endoscopic Unit, Faculty of Medicine, Zagazig University during the period from December 2010 to April 2013.

The study included 105 patients scheduled to undergo total abdominal hysterectomy with or without bilateral salpingoophorectomy who were divided into 3 groups:

Group $\boldsymbol{A}$ (35 patients) minilaparotomy hysterectomy using conventional sutures.

Group B (35 patients) minilaparotomy hysterectomy using bipolar vessel sealing system (Ligasure).

Group $\boldsymbol{C}$ (35 patients) laparoscopic hysterectomy using Ligasure.

Method of intervention was adopted according to patient desire. Appropriate informed consent was obtained from the patient after a thorough explanation of the planned operation, its potential risks and benefits.

Inclusion criteria:

All patients were scheduled to undergo hysterectomy for a benign uterine lesion such as:

- Leiomyoma.

- $\quad$ Endometrial hyperplasia.

- DUB unresponding to medical treatment.

- Suspected adenomyosis.

\section{Exclusion criteria:}

- Marked cardiopulmonary disease precluding Trendlenburg position.

- Any suspected or confirmed malignancy in the uterus.

- Uterine size > 12 weeks gestation.

- Uterine immobility due to previous laparotomies, pelvic endometriosis.

- Adnexal masses.

Full history taking, gynecologic examination and ultrasound evaluation were done to all patients.

The following data were collected from every patient in all groups:

- Age.

- BMI.

- Operative time.

- Intraoperative blood loss during laparotomy with the soaked towel meaning about $150 \mathrm{cc}$ blood loss and the soaked sponge meaning $5 \mathrm{cc}$. During laparoscopy the amount of blood loss was estimated by the amount in the suction container after subtracting the amount of fluid used for washing. Hemoglobin deficit 12 hours postoperatively was done for all patient groups.

Any intraoperative complications.

- $\quad$ Time to resumption of bowel movement.

- $\quad$ Pain score 12 hours postoperatively according to revised face pain scale .

Length of hospital stay.

Post-operative complications.

General anesthesia is used and prophylactic antibiotics are given in the form of 2 grams cefotaxime which is repeated as one gram 12 hours after surgery.

In the laparoscopy group, the operating table is designed to allow a deep Trendlenburg position. The patients are placed in padded Allen stirrups to provide good support and positioning. After thighs are slightly abducted, semiflexed, a uterine manipulator is fixed. In our study, Clermont-Ferrand manipulator is used (figure 1).

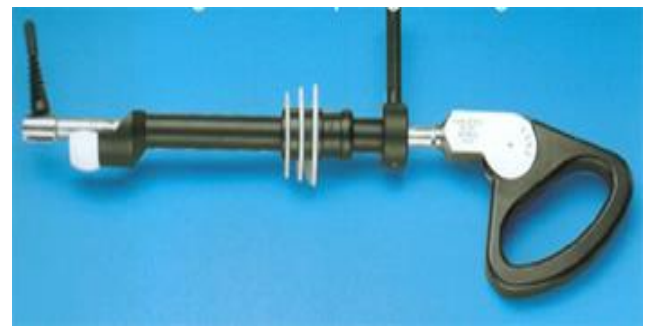

Figure (1): Clermont-ferrand manipulator

\section{Surgical technique:}

Groups A (minilaparotomy hysterectomy with conventional sutures) and B (minilaparotomy hysterectomy with Ligasure):

After general anesthesia, a Foley catheter is fixed within the bladder and the patient is put in Trendlenburg position.

A small transverse incision (4 cm in length) is made into the pubic hairline including skin and subcutaneous fat until reaching the anterior rectus fascia.

The fat is cleared from the midline superiorly and inferiorly to expose approximately $5-6 \mathrm{~cm}$ of fascia in the vertical axis. The anterior rectus fascia is cut in a Transverse (or) sometimes vertical (modified Kustner incision). The rectus muscles are separated, exposing the fascia transversalis and the peritoneum. The peritoneum is entered digitally or by scissors.

The uterus and the adnexa are brought outside the abdominal wall using the index and middle fingers as well as the thumb of the left 
hand and sometimes via either a strong traction suture in the uterine fundus or two long Kocher clamps lateral to the corpus. Two long, narrow abdominal retractors are used. Packing of the intestine is done.

The round ligaments are secured and cut at its middle part using either clamps and sutures (group A) or Ligasure (group B). This incision is directed inferiorly into the first 1 to $2 \mathrm{~cm}$ of the broad ligament.The tented anterior leaf is incised sharply, with the line of incision curving inferomedially to the level of the vesicouterine fold. Similarly, the posterior leaf of the broad ligament is opened. The incision extends inferomedially toward uterosacral ligaments.

Infundibulopelvic ligaments are secured and cut using either clamps and sutures (group A) or Ligasure (group B).

To mobilize the bladder, a hand can be wrapped around the uterus and a thumb is used to exert gentle pressure under the bladder and against the uterine surface inferiorly toward the vagina.

After skeletonization, The uterine vessels are either clamped horizontally across the vertical axis it then, divide and ligated (group A) or secured at two levels via Ligasure which is put also horizontally across the vertical axis of the vessels followed by cutting by the same instrument (group B).

The cardinal and uterosacral ligaments are divided as near as possible to the uterus via clamps and sutures (group A) or ligasure (group B).

The specimen is cut away from vaginal cuff which is closed via open cuff or closed cuff techniques.

The abdominal wall is closed as usual with the pelvic and parietal peritoneum unclosed and the rectus muscles are approximated in the midline via 2 to 3 interrupted sutures. The rectus sheath was then closed with vicryl No.2 running sutures. Subcutaneous fat was closed only if it is > $2 \mathrm{~cm}$ thickness using Vicryl No.2/0 sutures. The skin was closed by applying subcuticular nonabsorbable polypropylene sutures.

Group C (laparoscopic group):

Some kind of bleeding control instrument such as, bipolar and unipolar electricity generator, Ligasure vessel sealing instrument (figure 2) is used.

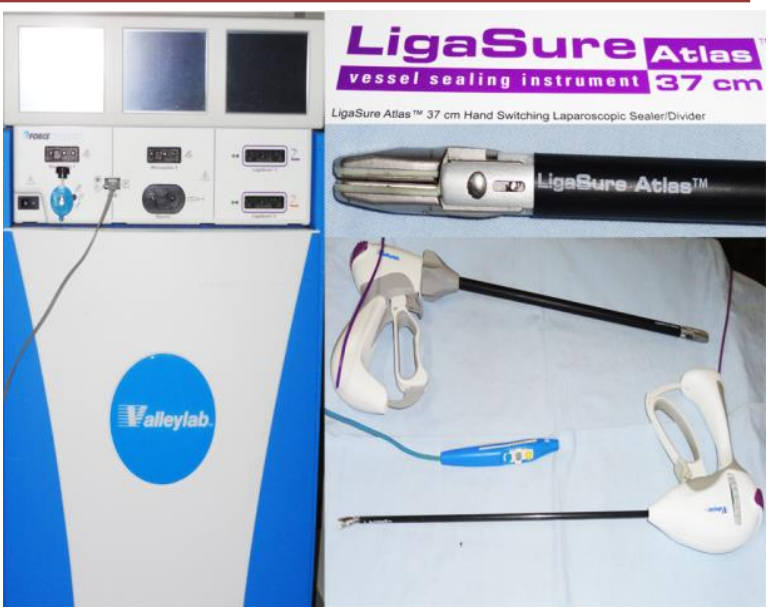

Figure (2): Ligasure vascular sealing system

A 10-12 $\mathrm{mm}$ trocar is placed through a vertical lower intraumbilical incision for insertion of the zero-degree telescope (Karl Storz, Tuttlingen, Germany). Two additional $5 \mathrm{~mm}$ trocars are then placed into the peritoneal cavity. This pair were placed lateral to the inferior epigastric vessels approximately two fingerbreadths above the pubis. the third one was $12 \mathrm{~mm}$ and put in the suprapubic region. In some cases, we used only two $(10 \mathrm{~mm})$ lateral ports without suprapubic one.

The round ligaments can easily be desiccated by using ligasure instrument. The uterus is deviated to the left by the manipulator and the assistant uses a grasper to place the round ligament under tension. The round ligament is coagulated and cut in the middle of the ligament with the coagulating instrument introduced from the ipsilateral side.

For women who want to preserve their ovaries, the uterine-ovarian ligaments and Fallopian tubes were sealed and cut medial to the ovary using ligasure atlas instrument $10 \mathrm{~mm}$. but if we want to remove the ovaries we cut the infundibelopelvic ligament itself taking care of the course of the ureter.

An assistant retroverts the uterus and pushes it cephalad using the manipulator. The upper junction of the vesico-uterine peritoneal fold is distinguished as a white line. Identification of the white line is important because, cephalad to the white line, the peritoneum is attached tightly to the uterus. Below this demarcation the peritoneum is loosely attached to the cervix and can be easily dissected away. The dome of the bladder is approximately $2 \mathrm{~cm}$ to $2.5 \mathrm{~cm}$ below the white line.

Using a grasper, the vesicouterine fold is placed under traction. A transverse incision is made just below the white line and the bladder is dissected away from the lower uterine segment 
and cervix using a peace of sponge on a grasper . In the right tissue plane, the dissection should be relatively bloodless. The middle band of loose connective tissue is the vesico-cervical ligament. This ligament does not contain blood vessels and can be easily divided. laparoscopic scissors with electroenergy can be used to coagulate any small incidental bleeders during dissection. The lateral bands of connection on both sides of the cervix are bladder pillars. The bladder pillars contain blood vessels and are desiccated prior to dissection.

The broad ligaments on both sides are opened downward and towards the cervix, skeletonizing the uterine vessels. Once the uterine vessels are skeletonized they can be sealed laterally using Ligasure atlas instrument $10 \mathrm{~mm}$. With meticulous dissection, the uterine vessels can be identified at the level of the ureteric canal as it crosses above the ureter. The uterine vessels can be secured medially as they enter the uterine body.

After dealing with the uterine artery, the manipulator is used to push the uterus to the left side during grasping and cutting the Mackenrodet's ligament on the right and vise versa. This is done using Ligasure instrument.

After that, we use the Valley laparoscopic hook or unipolar hook to open the vagina, we open the vagina anteriorly against the hard part of the manipulator and we move it laterally and posteriorly carefully to open the cuff laterally, then posteriorly. For fear of loss of pneumoperitoneum, we use a big sponge in a glove to pack the vagina tightly and surround the manipulator. Then, we remove the uterus vaginally. The surgeon put it in the opening and by a Vulsellum forceps we catch it vaginally and remove it.

After removing the uterus, we do underwater examination and for this purpose, we insufflate the peritoneum again, then irrigate the peritoneal cavity with $1000-1500 \mathrm{cc}$ saline, and inspect carefully all the pedicles and the vaginal vault, any bleeding point was controlled using bipolar forceps or Ligasure instrument. The vault is either left open or closed laparoscopically and sometimes vaginally.

\section{Statistical analysis:}

Statistical analysis was performed using the Statistical Package for Social Science for Windows (SPSS, Inc., Chicago, IL) version 14. A $\mathrm{P}$ value $<0.05$ was considered statistically significant.

Independent $\mathrm{t}$ test was used for statistical analysis, Variables with normal distribution were expressed as mean and 95\% CI \pm standard deviation.

\section{RESULTS}

There was no significant difference in age between the 3 groups, while in the laparoscopic group, the mean Body Mass Index (BMI) was significantly higher than the other groups (table 1).

the 3 groups were nearly similar regarding the indications of hysterectomy with fibroid uterus being the most common indication in all groups (table 2)

There was a significant difference between the 3 groups in the mean operative time with conventional minilaparotomy hysterectomy being faster (84.7 \pm 9.9 minutes) than laparoscopic hysterectomy $(94.8 \pm 16$ minutes $)$. The use of ligasure in minilaparotomy (group B) led to significant shortening of the operative time (55.3 \pm 7.8 minutes) than other groups.

The mean duration until resumption of intestinal sounds was significantly shorter in laparoscopic group $(12.3 \pm 2.5$ hours $)$ than both minilaparotomy groups(table 3 ).

The mean amount of blood loss and hemoglobin deficit were significantly lower in minilaparotomy with ligasure group in comparison to laparoscopic group which in turn was lower than conventional minilaparotomy group (table 4).

The mean duration of hospital stay showed no significant difference between minilaparotomy with ligasure group $(20.7 \pm 2.5$ hours $)$ and laparoscopic group (19.3 \pm 6 hours)., but both are significantly shorter in hospital stay than conventional minilaparotomy hysterectomy (31.8 \pm 5.7 hours).

There was a significant difference in the pain scores among the three groups with the highest pain score being observed in group A (5.2 $\pm 1.1)$ versus groups $\mathrm{B}(3.8 \pm 1.6)$ and $\mathrm{C}(3.7 \pm$ 1.2)table(5).

There was no statistically significant difference in the incidence of intraoperative or postoperative complications. Regarding intraoperative complications, There was two cases in group C (laparoscopic group) who experienced intraoperative bleeding without the need for blood transfusion. There was only one case of bladder injury which occurred in group C (laparoscopic group). During pushing the bladder flap downwards, the injury occurred and laparoscopic repair was done.

Postoperative complications in the form of stitch sinus was noted in one patient in group A while seroma formation was noted in 3 patiens in group $\mathrm{A}$ and 2 patients in group B. 
No complications was noted in $31(88.6 \%)$ B and 32(91.5\%) patients in group C.(table 6). patients in group A, 33(94.3\%) patients in group

Table (1): The preoperative demographic data for all of our patients

\begin{tabular}{cccccc}
\hline & A & B & C & F & P \\
\hline Age (years) & \multicolumn{5}{c}{0.41} \\
\hline Mean \pm SD & $50.5 \pm 4.4$ & $49.2 \pm 3.8$ & $50.2 \pm 4.4$ & 0.87 & 0.41 \\
\hline Range & $43-60$ & $43-59$ & $42-58$ & & \\
\hline BMI $\left(\mathbf{k g} / \mathbf{m}^{2}\right)$ & & & & \\
\hline Mean \pm SD & $28.1 \pm 2.2$ & $27.7 \pm 2.2$ & $29.7 \pm 2.6$ & 6.6 & \\
\hline Range & $23.2-32.2$ & $23-31.3$ & $23-34.95$ & & \\
\hline
\end{tabular}

Table (2): Indications for hysterectomy

\begin{tabular}{ccccccc}
\hline & \multicolumn{2}{c}{ A } & \multicolumn{2}{c}{ B } & \multicolumn{2}{c}{ C } \\
\cline { 2 - 7 } & No & \% & No & \% & No & \% \\
\hline Fibroid & 17 & 48.6 & 17 & 48.6 & 18 & 51.4 \\
\hline Adenomyosis & 4 & 11.4 & 5 & 14.3 & 4 & 11.4 \\
\hline DUB & 6 & 17.1 & 7 & 20 & 2 & 5.7 \\
\hline Endometrial hyperplasia & 7 & 20 & 6 & 17.1 & 9 & 25.7 \\
\hline Vesicular mole & 1 & 2.9 & 0 & 0 & 1 & 2.9 \\
\hline Endometrial polyp & 0 & 0 & 0 & 0 & 1 & 2.9 \\
\hline
\end{tabular}

Table (3): Operative time and intestinal sounds

\begin{tabular}{cccccc}
\hline & A & B & C & F & P \\
\hline Operative time (minutes) & & & & & \\
\hline Mean \pm SD & $84.7 \pm 9.9$ & $55.3 \pm 7.8^{*}$ & $94.8 \pm 16$ & 38.5 & $<0.001$ \\
\hline Range & $65-105$ & $45-75$ & $65-135$ & & \\
\hline Intestinal sounds (hours) & & & & \\
\hline Mean \pm SD & $17.4 \pm 1.9$ & $16.5 \pm 1.76$ & $12.3 \pm 2.5^{+}$ & 52.1 & $<0.001$ \\
\hline Range & $13-21$ & $12-18$ & $9-20$ & & \\
\hline
\end{tabular}

*Group B versus $\mathrm{A}$ and $\mathrm{C}$

${ }^{+}$Group $\mathrm{C}$ versus $\mathrm{A}$ and $\mathrm{B}$

Table (4): Blood loss and $\mathrm{Hb}$ deficit

\begin{tabular}{cccccc}
\hline \multicolumn{1}{c}{ A } & B & C & F & P \\
\hline Blood loss (ml) & & & & & \\
\hline Mean \pm SD & $130.3 \pm 54.4$ & $99.1 \pm 30.8^{*}$ & $136.6 \pm 6.6$ & 12.3 & $<0.001$ \\
\hline Range & $50-250$ & $50-150$ & $50-300$ & & \\
\hline Hb deficit (gm/dl) & & & & & \\
\hline Mean \pm SD & $0.68 \pm 0.25^{+}$ & $0.49 \pm 0.2^{*}$ & $0.84 \pm 0.5$ & 9.64 & $0.0019^{*}$ \\
\hline Range & $0.2-1.2$ & $0.2-0.9$ & $0.2-2$ & & \\
\hline
\end{tabular}

$*$ Group B versus $\mathrm{A}$ and $\mathrm{C}$

${ }^{+}$Group A versus C 
Table (5): Hospital stay and pain score

\begin{tabular}{ccccccc}
\hline \multicolumn{1}{c}{ A } & B & C & F & p \\
\hline Hospital stay (hours) & & & & & \\
\hline Mean \pm SD & $31.8 \pm 5.7^{*}$ & $20.7 \pm 2.5$ & $19.3 \pm 6$ & 31.3 & $<0.001$ \\
\hline Range & $24-48$ & $18-26$ & $15-48$ & & \\
\hline Pain score & & & & & \\
\hline Mean \pm SD & $5.2 \pm 1.1^{*}$ & $3.8 \pm 1.6$ & $3.7 \pm 1.2$ & 11.49 & $<0.001$ \\
\hline Range & $4-8$ & $2-6$ & $2-6$ & & \\
\hline
\end{tabular}

*Group A versus B and C

Table (6): Complications

\begin{tabular}{|c|c|c|c|c|c|c|c|c|}
\hline & \multicolumn{2}{|c|}{$\mathbf{A}$} & \multicolumn{2}{|c|}{ B } & \multicolumn{2}{|c|}{$\mathbf{C}$} & \multirow{2}{*}{$\mathbf{X}^{2}$} & \multirow{2}{*}{$\mathbf{P}$} \\
\hline & No & $\%$ & No & $\%$ & No & $\%$ & & \\
\hline No complications & 31 & 88.6 & 33 & 94.3 & 32 & 91.5 & 2.26 & 0.32 \\
\hline Seroma & 3 & 8.6 & 2 & 5.7 & 0 & 0 & 2.94 & 0.22 \\
\hline Stitch sinus & 1 & 2.8 & 0 & 0 & 0 & 0 & 2.02 & 0.36 \\
\hline Bladder injury & 0 & 0 & 0 & 0 & 1 & 2.8 & 4.08 & 0.13 \\
\hline IntraoperativeBleeding & 0 & 0 & 0 & 0 & 2 & 5.7 & 4.08 & 0.13 \\
\hline
\end{tabular}

\section{DISCUSSION}

Hysterectomy is one of the commonest gynecologic procedures.It ranks second only to cesarean section, with approximately 600,000 women undergoing this procedure annually in the United States for various indications ${ }^{(9)}$. Despite the introduction of advanced laparoscopic techniques, the vast majority of hysterectomies are still approached by either a vaginal $(23 \%)$ or abdominal route $(63 \%)$ while only $9.9 \%$ are done laparoscopically $^{(\mathbf{1 0})}$.

Each approach has its advantages, disadvantages, and its limitations. The laparoscopic approach has been widely accepted as a better alternative to standard laparotomy.

Most studies compared the laparoscopic approach to either the standard laparotomy approach ,the vaginal route or both. In this study, we tried to investigate the benefits of using Ligasure as a laparoscopic equipment to be able to do the hysterectomy procedure through a smaller abdominal incision that is minilaparotomy.

We compared conventional minilaparotomy (group A) and minilaparotomy using ligaSure (group B) with the laparoscopic hysterectomy (group C).

In group A, the results have shown that this method is inferior to the other methods with respect to blood loss,hemoglobin deficit, pain scoring ,return of bowel movements , and hospital stay. Inspite of the fact that the operative time was less than that in the laparoscopy group ,the inconvenience of the surgeons throughout the operation should be emphasized because of the small incision.

The mean operative time for conventional minilaparotomy $(84.7 \pm 9.9)$ was similar to that reported by Hoffman and Lynch ${ }^{(\mathbf{1 1 )}}$ (84 minutes). On the other hand, it was longer than that reported by Chalkoo et al. ${ }^{(1)}$, Sharma et al. ${ }^{(7)}$, and Gungor et al. ${ }^{(12)}$ where the operative time was 30 (20-63) minutes, 41 (30-90) minutes and 45 (30-90) minutes respectively. The operative time ranged from 65 to 105 minutes with 5 cases being longer than 90 minutes. In these cases the BMI was more than $30 \mathrm{~kg} / \mathrm{m}^{2}$ with the other patient selection criteria similar to other groups.

In this group ,the mean blood loss was $130.3 \pm 54.4 \mathrm{ml}$ and the hemoglobin deficit was 
$0.71 \pm 0.25 \mathrm{gm} / \mathrm{dl}$. The mean blood loss was more than that reported by Chalkoo et al. ${ }^{(\mathbf{1})}(20 \mathrm{ml})$ and that reported by Gungor et al. ${ }^{(\mathbf{1 2})}(100 \mathrm{ml})$, while it was less than that reported by Mahendru et al. $^{\text {(13) }}$ (240 ml) and Sharma et al. ${ }^{(7)}(240 \mathrm{ml})$. The wide range of blood loss in this group (50 -250 $\mathrm{ml}$ ) can be attributed to the adhesions found in some cases with previous pelvic surgery.

The mean duration of return of intestinal sounds $(17.4 \pm 1.9$ hours $)$ and the mean duration of hospital stay in conventional minilaparotomy group (31.8 \pm 5.7 hours) were significantly longer than that in other groups. Also, the mean pain score was higher $(5.2 \pm 1.1)$ and sometimes the patients needed further analgesia. These results are comparable to that reported by Sharma et al. ${ }^{(7)}$ and Mahendru et al. ${ }^{(13)}$.

Regarding group B, all the parameters of comparison were superior to that in group A.The recent trends towards minimally invasive surgery has lead to the continuous trials of refinement in laparotomy techniques .

The use of LigaSure in this group led to significant shortening of the operative time with the mean time was $55.3 \pm 7.8$ minutes in comparison to $(84.7 \pm 9.9$ minutes $)$ in group $\mathrm{A}$ and (94.8 \pm 16 minutes) in group $\mathrm{C}$. This is shorter than that reported by Royo et al. ${ }^{(\mathbf{1 4})}$ using the same technique with nearly the same inclusion and exclusion criteria $\quad$ [73.4 (67.8-78.9) minutes].

The mean amount of blood loss in group B was significantly lower $(99.1 \pm 30.8 \mathrm{ml})$ than conventional minilaparotomy group $(130.3 \pm 54.4$ $\mathrm{ml})$ and the laparoscopy group (128.6 \pm 6.6 $\mathrm{ml}$ ).As a consequence of blood loss, the mean hemoglobin deficit 12 hours after surgery was significantly lower $(0.49 \pm 0.2 \mathrm{gm} / \mathrm{dl})$ than conventional minilaparotomy group $(0.71 \pm 0.25$ $\mathrm{gm} / \mathrm{dl})$ and laparoscopic group $(0.68 \pm 0.5 \mathrm{gm} / \mathrm{dl})$.

These results are different form those reported by Royo et al. ${ }^{(14)}$ who reported that laparoscopy group had less hemoglobin deficit $(1.8 \mathrm{gm} / \mathrm{dl})$ than minilaparotomy hysterectomy with ligasure group $(2.1 \mathrm{gm} / \mathrm{dl})$.

The mean pain score in group B $(3.8 \pm 1.6)$ was not statistically different from that in the laparoscopy group $(3.7 \pm 1.2)$. This could be attributed to the use of few instruments without the need for large or self retaining abdominal wall retractors .These results are similar to that reported by Royo et al. ${ }^{(14)}$ who reported no difference in pain scores between minilaparotomy hysterectomy (using ligasure) or laparoscopic hysterectomy.

The mean duration of return of intestinal sounds ( $16.5 \pm 1.76$ hours) was longer than that in laparoscopy group while the mean duration of hospital stay $(20.7 \pm 2.3$ hours $)$ was not statistically different between both groups.

In group $\mathrm{C}$ all the advantages of laparoscopic surgery were obvious; the view was very clear.With proper manipulations, accessibility to various pelvic structures was easy with proper inspection of all pelvic recesses . Adhesiolysis of attachment of bowel or any other structures to the genital organs was achieved before hysterectomy. Although dealing with big uteri is known to be troublesome ,proper uterine manipulation from below facilitated the laparoscopy route.

The mean operative time in group $\mathrm{C}$ was $94.8 \pm 16$ minutes . It deserves mentioning that the operative time in laparoscopy group showed considerable shortening with repetition of cases and build up of experience starting by 120-135 minutes in the first 6 cases and declining to reach 70-75 minutes in the last 5 cases.

The mean operative time was shorter than that reported by Royo et al. ${ }^{(14)}$, Drahonovsky et al. ${ }^{(15)}$, and Roy et al. ${ }^{(\mathbf{1 6})}$ which were 159.3 (140.2178.5) minutes, 111 (55-180) minutes], and 100 (75-150) minutes respectively.

One of the inherent aspects of laparoscopic surgery is the proper hemostasis necessary for good visualization .The mean amount of blood loss in the laparoscopic group was (128.6 \pm 6.6 $\mathrm{ml}$ ) which is less than that reported by Drahonovsky $^{(\mathbf{1 5})}(184 \mathrm{ml})$ and Roy et al. ${ }^{(\mathbf{1 6})}$ (250 $\mathrm{ml})$. It is to be mentioned that 2 cases of intraoperative bleeding occurred in laparoscopy group.In these 2 cases ,the descending cervical branch slipped and this necessitated one more application of ligasure bipolar vessel sealing system without the need for conversion to laparotomy or blood transfusion.

The mean duration until return of intestinal sounds was significantly shorter in laparoscopic hysterectomy group $(12.3 \pm 2.5$ hours $)$ than group A $(17.4 \pm 1.9$ hours $)$ and group B $(16.5 \pm 1.76$ hours). These results are similar to that reported by Sharma et al. ${ }^{(7)}$ and Mahendru et al. ${ }^{(13)}$. This significant difference could be attributed to less intestinal handling ,less exposure to room temperature with consequent tissue dryness ,non use of laparotomy pads , and less electrolyte disturbance.

The mean duration of hospital stay in laparoscopic hysterectomy group was $(19.3 \pm 6$ hours) being significantly shorter than group A and similar to group B $(20.7 \pm 2.3$ hours $)$. This significant difference was noticed in most of the studies in the literature. However, it can be noticed that the mean hospital stay in previous 
studies is significantly longer. The mean hospital stay for minilaparotomy hysterectomy with ligasure group was 3.9 days in Royo et al. ${ }^{(14)}$ study compared to 3.2 days for laparoscopic hysterectomy.

Throughout the study, it was our trend to discharge patients in the next morning after being sure of sound uneventful postoperative course. This resulted in a dramatic fall in duration of hospital stay from 2-4 days to 12-17 hours. There were no major or minor intraoperative or postoperative complications except one case of bladder injury. These data favour adoption of this approach. It is to be mentioned that Chalkoo et al. ${ }^{(1)}$ confirmed in their study that this strategy is safe and only the complicated cases need prolonged hospital stay. There is no doubt that reduced hospital stay has its implications on the cost savings to medical systems.

The mean pain score was $(3.7 \pm 1.2)$ which is not statistically different from that in group B $(3.8 \pm 1.6)$.

The complication rate in all groups was very low. No intraoperative complications were encountered in group A or B .only one case of bladder injury was encountered in the first case of group $\mathrm{C}$ during sharp dissection. The injury was immediately recognized and repaired. Short and long term follow up of this case was unremarkable.

The postoperative complication rate was low overall the study. In conventional minilaparotomy hysterectomy group, there was a case of stitch sinus and 3 cases of seroma formation, while seroma occurred in 2 cases of minilaparotomy hysterectomy with ligasure group. All those patients underwent frequent dressing with sound healing without the need for readmission or additional antibiotics.

The complication rate in our study was comparable to that reported by Chalkoo et al. ${ }^{(1)}$; Mahendru et al. ${ }^{(13)}$ and Royo et al. ${ }^{(14)}$; while it was less than that reported by Sharma et al. ${ }^{(7)}$ and Hoffman and Lynch ${ }^{(11)}$.

\section{CONCLUSION}

In comparison to conventional minilaparotomy, laparoscopic hysterectomy took slightly longer operative time with the same intraoperative blood loss but ess duration until resumption of intestinal sounds, less postoperative pain scoring and less hospital stay.

Minilaparotomy hysterectomy with ligasure may be a suitable effective alternative to laparoscopic hysterectomy, combining the technical advantages of laparotomy (e.g. short learning curve than laparoscopy) and the convalescent advantages of laparoscopic surgery (low morbidity, short hospital stay and good cosmetic results). Thus, it can be ideal in areas who lack high laparoscopic experience or facilities

Minilaparotomy hysterectomy and laparoscopic hysterectomy are considered safe in terms of intraoperative and postoperative complications.

\section{REFERENCES}

1- Chalkoo M, Ahangar S, Durrani AM, et al. Minilaparotomy hysterectomy-revisited with new concepts and technical modifications. International Journal of Surgery 2011; 9: 404-409.

2- Campbell, E.S.; Xiao, H. and Smith, M.K. Types of hysterectomy: Comparison of characteristics, hospital costs, utilization and outcome. J Reprod Med 2003; 48(12): 943-9.

3- Fanfani, F.; Fagotti, A. and Longo, R.; et al. Minilaparotomy in the management of benign gynecologic disease. European Journal of Obst. Gynecol. and Reprod. Biology 2005; 119: 232236.

4- Panici, P.B.; Zullo, M.A.; Angioli, R. and Muzii, L. Minilaparototomy hysterectomy: A valid option for the treatment of benign uterine pathology. Europ. J. Obstet. Gynecol. Reproductive Biology 2005; 119: 228-231.

5- Munro, M.G.; Brill, A.I. and Parker, W.H. Gynecologic endoscopy. In: Berek and Novak's Gynecology (14 ${ }^{\text {th }}$ edition) 2007; 2(21): 749-804.

6- Pejovic, T. and Nezhat, F. Laparoscopic management of adnexal masses: The opportunities and the risk. Ann Ny Acad Sci 2001; 943: 255-68.

7- Sharma JB, Wadhwa L, Malhotra M, et al. Minilaparotomy versus conventional laparotomy for abdominal hysterectomy: A comparative study. Indian J. Med. Sci. 2004; 58: 196-202.

8- Pelosi MA II and Pelosi MA III. Pelosi minilaparotomy hysterectomy: Effective alternative to laparoscopy and laparotomy. J Am Assoc Gynaecol Laparosc 2003.

9- Farquhar, C. and Steiner, C.A. Hysterectomy rates in the United States 1990-1997. Obstet Gynecol 2002; 99: 229-34.

10- Advincula AP and Falcone T. Minimally invasive gynecologic surgery ;Nezhat operative gynecologic laparoscopy and hysteroscopy $3^{\text {rd }}$ ed 2008; 23;23.2(567-576).

11- Hoffman MS and Lynch CM. Minilaparotomy hysterectomy. Am J Obstet Gynecol 1998; 179: 316-320.

12- Gungor M, Ortac F, Cengiz B, et al. Laparoscopic versus microlaparotomy or modern minilaparotomy hysterectomy. Journal of Gynecologic Surgery 1999; 15(4).

13- Mahendru R, Malik S and Mittal A. Minilaparotomy hysterectomy: A worthwhile alternative. J. Obstet. Gynaecol. Res. 2011; 37(4): 305-312.

14- Royo P, Alcazar JL, Manero MG, et al. The value of minilaparotomy for total hysterectomy for 
benign uterine disease: A comparative study with conventional Pfannenstiel and laparoscopic approaches. International Archives of Medicine 2009; 2: 11 .

15- Drahonovsky J, Haakova L, Otcenasek M, et al. A prospective randomized comparison of vaginal hysterectomy, laparoscopically assisted vaginal hysterectomy, and total laparoscopic hysterectomy in women with benign uterine disease. European Journal of Obstetrics and Gynecology and Reproductive Biology 2010; 148: 172-176.

16- Roy KK, Goyal M, Singla S, et al. A prospective randomized study of total laparoscopic hysterectomy, laparoscopically assisted vaginal hysterectomy and non-descent vaginal hysterectomy for the treatment of benign diseases of the uterus. Arch Gynecol Obstet 2010. 